\title{
Malaria Prevalence in a Low Transmission Area, Jazan District of Southwestern Saudi Arabia
}

\author{
Yousry Hawash ${ }^{1,2, *}$, Khadiga Ismail ${ }^{1,3}$, Khalaf Alsharif', Walaa Alsanie ${ }^{1}$ \\ ${ }^{1}$ Clinical Laboratories Sciences Department, College of Applied Medical Science, Taif University, Taif, Saudi Arabia; ${ }^{2}$ Parasitology Department, \\ National Liver Institute, Menoufia University, Menoufia, Egypt; ${ }^{3}$ Parasitology Department, Faculty of Medicine, Ain-Shams University, Cairo, Egypt
}

\begin{abstract}
Detailed description of malaria in low transmission areas is crucial for elimination. The current study aimed to provide a comprehensive description for malaria transmission in Jazan, a low transmission district, southwestern Saudi Arabia. Patients at a tertiary care hospital were recruited in our study between August 2016 and September 2018. Malaria diagnosis was performed through a species-specific nested polymerase chain reaction (nested PCR), microscopy and Paramax- $3^{\mathrm{TM}}$ rapid detection test (RDT). Malaria was detected in 30 patients by the PCR, with point prevalence of $10.9 \%$. Of these malaria infections, $80 \%$ was imported, $26.6 \%$ was asymptomatic and $23.3 \%$ was sub-microscopic. Malaria was reported throughout the year, with February/March and September/October peaks. Infection was significantly more in males than in females $(P=0.01)$. Likewise, infections were detected more in febrile than in non-febrile patients $(P=0.01)$. Adult aged 15-24 years, fever and travel were identified as high-risk factors. Malaria was primarily attributed to Plasmodium falciparum mono-infections, followed by $P$. vivax mono-infections and lastly to falciparum/vivax mixed infections accounting $76.6 \%, 16.6 \%$, and $6.6 \%$ of PCR-confirmed malaria cases, respectively. The nested PCR was superior to the smear microscopy (sensitivity $76.6 \%$; specificity $100 \%$ ) and the RDT (sensitivity $83.3 \%$, specificity $94.2 \%$ ). The overall percent agreement between microscopy and the RDT was $92.7 \%$ (kappa $=0.63$ ). High proportion of imported malaria including sub-microscopic and sub-patent cases were described. We suggest that incorporation of molecular tool into the conventional malaria diagnosis is beneficial in Jazan district.
\end{abstract}

Key words: Malaria diagnosis, nested PCR, Paramax-3 $3^{\mathrm{TM}}$, sub-microscopic/asymptomatic infections, Saudi Arabia

\section{INTRODUCTION}

The burden of malaria infection has been reduced over the past decades in many countries. Nonetheless, malaria remains an important parasitic disease in humans. To date, there are 5 Plasmodium species able to cause malaria in humans: P. falciparum, P. vivax, P. malariae, P. ovale and P. knowlesi [1]. Subspecies of $P$. ovale also infect humans [2]. Human infection is often transmitted through the skin bite with the female Anopheles mosquito while taking a blood meal. Globally, the majority of the malaria cases were attributed to either $P$. falciparum or $P$. vivax species. Infections attributed to the other Plasmodium species and/or subspecies are relatively low [3].

Malaria symptoms vary based on the level of host immunity

- Received 13 November 2018, revised 24 March 2019, accepted 3 May 2019

*Corresponding author (yousryhawash@gmail.com)

(c) 2019. Korean Society for Parasitology and Tropical Medicine

This is an Open Access article distributed under the terms of the Creative Commons Attribution Non-Commercial License (http://creativecommons.org/licenses/by-nc/4.0) which permits unrestricted non-commercial use, distribution, and reproduction in any

medium, provided the original work is properly cited. and the pattern of transmission across countries. In the high transmission areas, people develop partial immunity render them capable to tolerate high levels of parasitaemia without symptoms owing to their constant exposure to infection. In contrast, in the low transmission areas, people develop malaria symptoms at low levels of parasitaemia [4]. Malaria diagnosis is usually carried out via traditional diagnostics (Light microscopy and rapid diagnostic tests [RDT]) [5]. Sensitive, quantifiable, high throughput, and field-friendly molecular diagnostic tools have been developed for malaria diagnosis [6].

In Saudi Arabia, malaria is confined to the southwestern parts of the country [7]. Malaria was constrained mostly to foci in Aseer and Jazan regions. In these locations, malaria is mostly imported, and the indigenous malaria is often recognized as low [8]. In areas where the malaria transmission is low, the asymptomatic malaria infections is high and tend to be sub-microscopic. The residual immunity developed in these settings, is believed to be the reason [9]. People with asymptomatic infections may develop symptoms in days or weeks after detection or persist sub-patent for months at unsteady levels of par- 
asitaemia [10]. These asymptomatic sub-microscopic infections constitute an important threat to malaria elimination through local transmission in the low transmission areas.

Jazan is a low transmission malaria district, southwestern Saudi Arabia. Few studies have been performed to describe malaria transmission in the region $[11,12]$. The current study aimed to provide an update for malaria transmission in Jazan. Our objectives were to identify the infection's pattern (indigenous, imported, asymptomatic, symptomatic, microscopic and sub-microscopic), to define the causative species and lastly to determine factors that contribute to transmission of malaria in the study's area. To maximize the sensitivity of malaria diagnosis, a species-specific nested polymerase chain reaction (nested PCR) was used in addition to the conventional diagnostics (microscopic examination and the RDTs). Taking the nested PCR results as a reference, the diagnostic performance of the microscopy and the RDT were also investigated in our study.

\section{MATERIALS AND METHODS}

\section{Study area}

Jazan (or Jizan) district carries the highest burden of malaria in Saudi Arabia, with approximately 1.5 million people living at risk of infection. Jazan is a high-altitude area 400-2,500 miles above the sea level. The province has set of factors that affect malaria control: the area is inhabited by a highly mobile residents, the area lies close to the southwestern part of Yemen where malaria is highly-endemic and a military conflict is still ongoing. Fig. 1 shows the map of Saudi Arabia including the study's area and the nearby cities. Rain in Jazan usually fall in August through October [13].

\section{Ethics statement, study design and population}

Ethical approval was obtained from the Institutional Review Boards at Taif University (TU-IRB). All participants and patients were informed by the study and signed consents were obtained according to the ethical standards. Prior to examination, relevant information was given to patients, including information about the sampling technique and the benefits of the study. Patients attending the outpatient clinic at King Fahd Central Hospital were invited to participate in the study. Children under 5 years old, pregnant women, patients taking antimalarial medications, and those presented with signs of severe complicated malaria infections were excluded from participation. Malaria screening was carried out between August 2016 and September 2018.

\section{Blood sampling and data collection}

The basic demographic features such as the patient's gender, age, residence and nationality were recorded. The axillary body temperature was measured, and fever was defined as a temperature exceeds $37.5^{\circ} \mathrm{C}$. History of a recent travel to a malaria-en-

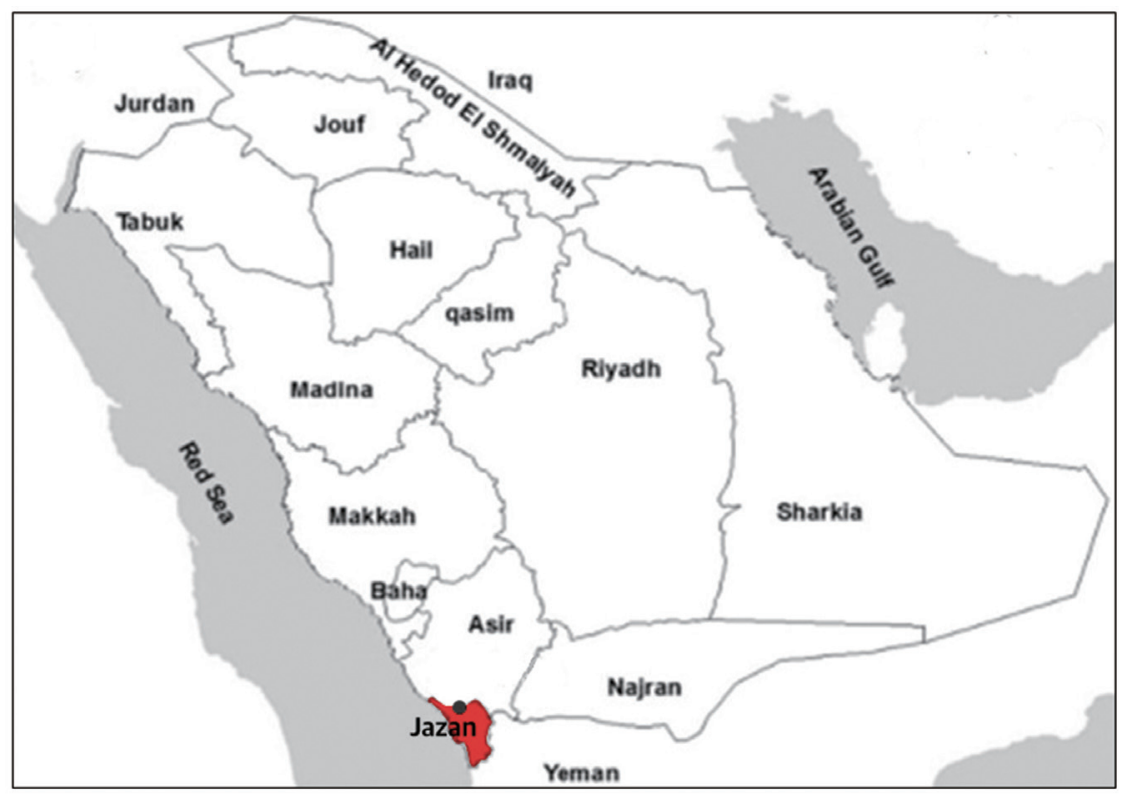

Fig. 1. Map of Saudi Arabia showing the district of Jazan. 
demic area(s) was also reported. The rapid detection test and blood smears were prepared on site using finger-prick blood, stored in separate boxes and transported to the laboratory. From the same prick, about $75 \mu \mathrm{l}$ blood was withdrawn via a $75 \mathrm{~mm}$ capillary tube, spotted on Whatman filter paper (Whatman International, Maidstone, UK) and left to dry at room temperature. Thoroughly dried blood samples were individually stored in plastic bags, transported to the laboratory, kept at $-20^{\circ} \mathrm{C}$ for molecular testing.

\section{Conventional diagnosis}

Giemsa stained blood films (thick and thin) were prepared from capillary blood (finger prick) and used for the microscopic detection of the Plasmodium stages in patient's blood, as described in a previous report [14]. Paramax- $3^{\mathrm{TM}}$ RDT (Zephyr Biomedicals, Goa, India) was used for malaria rapid diagnosis. The test detects the histidine-rich protein 2 antigen of $P$. falciparum, the lactase dehydrogenase antigen for $P$. vivax and the aldolase antigen for Plasmodium spp. The test was performed and interpreted according to the manufacturer's instructions.

\section{Molecular diagnosis}

Genomic DNA sample was purified from a quarter of a filter paper blood spot using the QIAamp Blood Mini Kit (Qiagen, Germantown, USA). Nucleic acid sequences of the mitochondrial cytochrome c oxidase III ( $\operatorname{cox} 3$ ) target gene were amplified with 2-round PCR [15]. The first round's primers were genusspecific while that for the second (nested) round were speciesspecific. Primers were synthesized by the VHBio (Gateshead, UK), dissolved in distilled water for stock preparation (100 $\mathrm{pmol} / \mu \mathrm{l}$ ) and kept at $-20^{\circ} \mathrm{C}$ till use. PCR reactions were set up in patches of ten samples, in Techne $e^{\mathrm{TM}} \mathrm{TC}-4000$ Thermocycler. PCR reagents with the final concentrations, used in our research, were closely similar to that of the published protocol [15]. The amplification products of the first round (940 bp) were examined on $0.8 \%$ agarose gel electrophoresis while that of the second round ( $87-233 \mathrm{bp}$ ) were analysed on $2 \%$ agarose.

\section{Data analysis}

The patients' data and laboratory tests results were collected, recorded and analysed using SPSS V. 21 software (SPSS, Chicago, Illinois, USA). Variations in the patients' demographic features and malaria prevalence among distinct age groups were assessed using the Chi-squared test. The diagnostic test's sensitivity (Se), specificity (Sp), negative predictive value (NPV) and positive predictive value (PPV) were calculated with the 95\% confidence intervals (CI) against the nested PCR test's results. The variable difference among the age groups with a $P$-value of less than 0.05 was considered statistically significant.

\section{RESULTS}

\section{Population description}

There were 321 patients agreed to participate in our study. Patients met the inclusion criteria $(n=275)$ were enrolled in the study. The description of study's population is stated in Table 1 . Most of the patients were females $(165 ; 60 \%)$ with mean age of $22.1 \pm 10.7$ years. The patients' age was ranged between 5 and 64 years, with a mean age of $23.5 \pm 13.19$ years and a median age of 21 years. Over $73 \%$ of the participants were below 25 years. Fifty-one per cent of patients were Saudi citizens and about $49 \%$ were non-Saudi immigrants. The majority of patients $(73 \% ; 221 / 275)$ reported a history of recent travel to malaria-endemic areas.

\section{Prevalence of malaria by PCR}

The target Plasmodium DNA was identified in 30 patients by the nested PCR. The point prevalence rate was 10.9\% (30/ 275). The malaria was attributed to $P$. falciparum mono-infections, $P$. vivax mono-infections and falciparum/vivax mixed infections in $76.6 \%$ (23/30), 16.6\% (5/30), and 6.6\% (2/30) of PCR-confirmed cases, respectively (Fig. 2A). No other species was detected in the study's population.

\section{Case description}

The malaria cases were reported all the year around, with

Table 1. Description of the 275's participants

\begin{tabular}{llc}
\hline Character & Category & Proportion (\%) \\
\hline Age groups (year) & $5-14$ & 22.9 \\
& $15-24$ & 49.8 \\
& $25-44$ & 18.6 \\
Fever & $\geq 45$ & 8.7 \\
& Yes & 54.5 \\
Gender & No & 45.5 \\
& Male & 40 \\
Residency & Female & 60 \\
\multirow{2}{*}{ Travel history } & Rural & 64 \\
& Urban & 36 \\
Nationality & Yes & 73 \\
& No & 27 \\
& Saudi & 51.3 \\
\hline
\end{tabular}



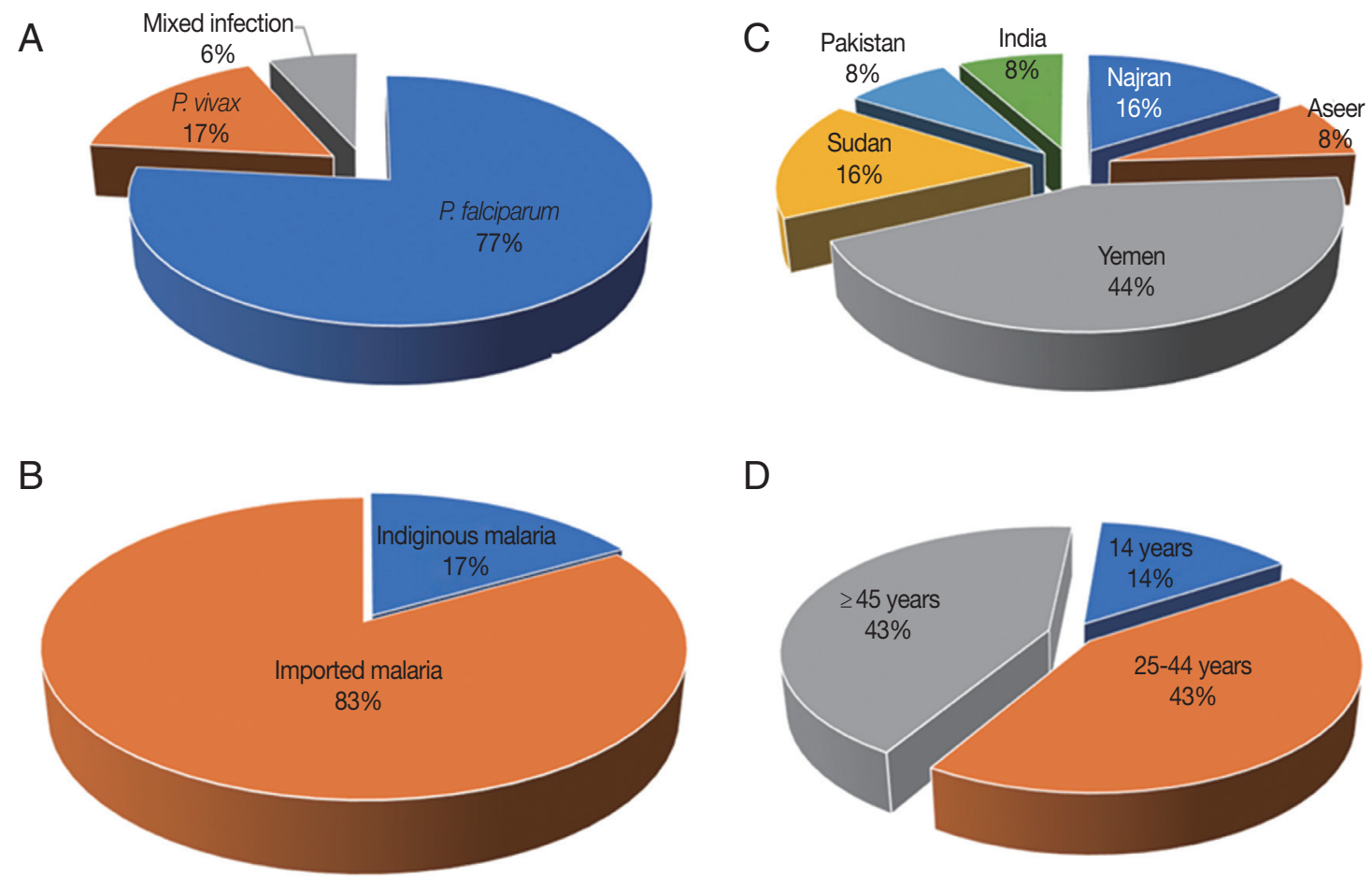

D

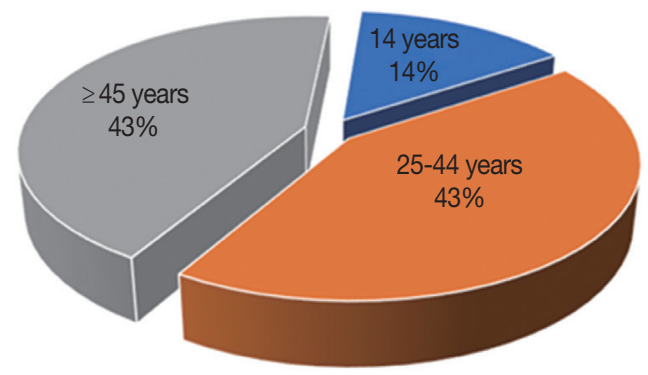

Fig. 2. Characteristics of the species distribution 30 malaria cases (A). (B) Proportions of imported and indigenous malaria in Jazan, 2016-2018. (C) Source countries/regions of imported malaria. (D) Age distribution of seven sub-microscopic malaria cases.

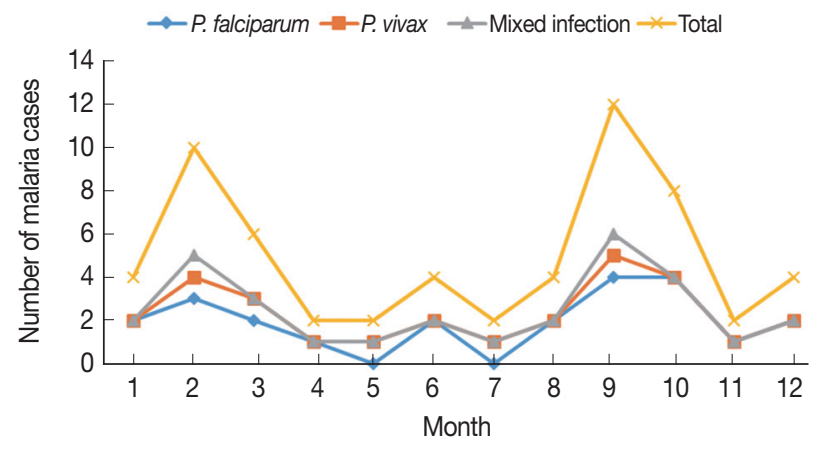

Fig. 3. Month-wise distribution of the reported malaria cases in Jazan, 2016-2018.

February-March and September-October evident peak periods, accounting 26.6\% (8/30) and 33.3\% (10/30) of the PCR-detected malaria cases, respectively (Fig. 3). Malaria infection was predominant $(53.3 \% ; 16 / 30)$ in patients aged 15 to 24 years, followed by children $5-14$ years $(26.6 ; 8 / 30)$ and patients over 24 years $(20 \% ; 6 / 30)$, with non-significant difference ( $P$-value $=0.6415)$ observed among the different age groups. The vast majority of malaria cases were detected in males $(60 \%$; $18 / 30$ ) and non-Saudi residents (63.3\%; 19/30). Also, most of malaria cases were described in those presented with fever
(73.3\%; 22/30), those reside rural areas $(80 \% ; 24 / 30)$ and those travelled to known malaria-endemic areas (80\%; 24/30). Significant difference was observed regarding infection transmission and the patient's gender $(P$-value $=0.0178)$ as well as the presence of fever $(P$-value $=0.0285)$ (Table 2$)$.

\section{Symptomatic versus asymptomatic malaria cases}

Malaria was confirmed in 22 febrile patients by the nested PCR. The point prevalence of symptomatic malaria was $14.6 \%$ (22/150). Patent malaria cases were attributed to $P$. falciparum mono-infections, $P$. vivax mono-infection and falciparum/vivax mixed infection in $90.9 \%(20 / 22), 4.5 \%(1 / 22)$, and $4.5 \%$ (1/22), respectively. On the other hand, 8 asymptomatic (i.e. sub-patent) malaria cases were picked up by the nested PCR (Table 3). The point prevalence asymptomatic malaria was $6.4 \%$ (8/125). The sub-patent malaria was attributed to $P$. falciparum mono-infections, $P$. vivax mono-infection and falciparum/vivax mixed infection in 37.5\% (3/8), 50\% (4/8), and $12.5 \%(1 / 8)$ of cases, respectively.

\section{Imported versus indigenous malaria cases}

Among the 30 malaria cases, 25 (83.3\%) had a recent travel 
Table 2. Characteristics of PCR-confirmed malaria cases

\begin{tabular}{|c|c|c|c|c|}
\hline Variable & Category & Positive (\%) & No. of cases & $P$-value \\
\hline Age group (year) & $\begin{array}{l}5-14 \\
15-24 \\
25-44 \\
\geq 45\end{array}$ & $\begin{array}{r}12.6 \\
11.6 \\
5.8 \\
12.5\end{array}$ & $\begin{array}{r}63 \\
137 \\
51 \\
24\end{array}$ & 0.6415 \\
\hline Fever & $\begin{array}{l}\text { Yes } \\
\text { No }\end{array}$ & $\begin{array}{r}14.6 \\
6.4\end{array}$ & $\begin{array}{l}150 \\
125\end{array}$ & $0.0285^{\star}$ \\
\hline Gender & $\begin{array}{l}\text { Male } \\
\text { Female }\end{array}$ & $\begin{array}{r}16.3 \\
7.2\end{array}$ & $\begin{array}{l}110 \\
165\end{array}$ & $0.0178^{*}$ \\
\hline Residency & $\begin{array}{l}\text { Rural } \\
\text { Urban }\end{array}$ & $\begin{array}{r}13.6 \\
6.0\end{array}$ & $\begin{array}{r}176 \\
99\end{array}$ & 0.053 \\
\hline Travel history & $\begin{array}{l}\text { Yes } \\
\text { No }\end{array}$ & $\begin{array}{r}11.9 \\
8.1\end{array}$ & $\begin{array}{r}201 \\
74\end{array}$ & 0.3659 \\
\hline Nationality & $\begin{array}{l}\text { Saudi } \\
\text { Non-Saudi }\end{array}$ & $\begin{array}{r}7.8 \\
14.2\end{array}$ & $\begin{array}{l}141 \\
134\end{array}$ & 0.0899 \\
\hline
\end{tabular}

*Statistically-significant.

Table 3. Distribution of symptomatic and asymptomatic malaria cases by age group

\begin{tabular}{|c|c|c|c|c|c|c|}
\hline \multirow{2}{*}{ Category } & \multirow{2}{*}{ Species } & \multicolumn{4}{|c|}{ Positive age group (year) } & \multirow{2}{*}{ Subtota } \\
\hline & & $5-14$ & $15-24$ & $25-44$ & $\geq 45$ & \\
\hline \multirow[t]{4}{*}{ Symptomatic $(n=150)$} & P. f mono-infection & 4.6 & 8.6 & 0 & 0 & 13.3 \\
\hline & P. $v$ mono-infection & 0.0 & 0.6 & 0 & 0 & 0.6 \\
\hline & $(P . f .+P . v$.$) Mixed$ & 0.0 & 0.6 & 0 & 0 & 0.6 \\
\hline & Subtotal & 4.6 & 10.0 & 0 & 0 & 14.6 \\
\hline \multirow[t]{4}{*}{ Asymptomatic $(n=125)$} & P. f mono-infection & 0.0 & 0.8 & 0.8 & 0.8 & 2.4 \\
\hline & P. $v$ mono-infection & 0.8 & 0.0 & 1.6 & 0.8 & 3.2 \\
\hline & $(P . f .+P . v$.$) Mixed$ & 0.0 & 0.0 & 0.0 & 0.8 & 0.8 \\
\hline & Total & 0.8 & 0.8 & 2.4 & 2.4 & 6.4 \\
\hline
\end{tabular}

P. f, Plasmodium falciparum; P. v., Plasmodium vivax.

history to malaria-endemic areas and thereby designated as imported malaria case, Fig. 2B. The imported malaria was reported in the study's setting with a point prevalence rate of $9.0 \%$ (25/275). Six malaria cases (5 P. falciparum mono-infections and one falciparum/vivax mixed infection) had visited other malaria-endemic Saudi areas like Aseer $(\mathrm{n}=2)$ and Najran $(\mathrm{n}=4)$. The remainder malaria cases (14 P. falciparum mono-infections and 5 P. vivax mono-infections) were migrants: 11 (57.8\%) from Yemen, 4 (21\%) from Sudan, 2 (10.5\%) from India and 2 (10.5\%) from Pakistan, Fig. 2C. By comparison, $16.6 \%$ (5/30) of the PCR-confirmed malaria cases had not left Jazan to malaria-endemic areas, and thereby designated as indigenous (i.e. autochthonous; native) malaria cases. The burden of native malaria in the study setting was $1.8 \%$ (5/275). The autochthonous malaria was ascribed to $P$. falciparum mono-infections and falciparum/vivax mixed infection in $80 \%(4 / 5)$ and $20 \%(1 / 5)$, respectively.

\section{Sub-microscopic malaria cases}

Of all PCR-confirmed malaria cases, 23 (76.6\%) were identified by the microscopic examination, with estimated point prevalence of $8.3 \%$ (23/275). Of these malaria cases, 22 (95.6\%) were patent (symptomatic) infections. Malaria cases, detected by microscopy, were attributed to $P$. falciparum monoinfections, P. vivax mono-infections and falciparum/vivax mixed infection in $86.9 \%$ (20/23), 8.6\% (2/23), and 4.3\% (1/23), respectively. Moreover, out of the 30 malaria cases detected by the PCR, 7 (23.3\%) were missed identification by microscopic examination (i.e. sub-microscopic). The estimated prevalence rate of sub-microscopic malaria in the study's population was $2.5 \%$ (7/275). Importantly, all the sub-microscopic malaria cases were sub-patent infections. Equally important, the submicroscopic malaria cases were attributed to P. falciparum mono-infections, P. vivax mono-infections and falciparum/vivax mixed infection in $42.8 \%(3 / 7), 42.8 \%(3 / 7)$, and $14.2 \%$ 


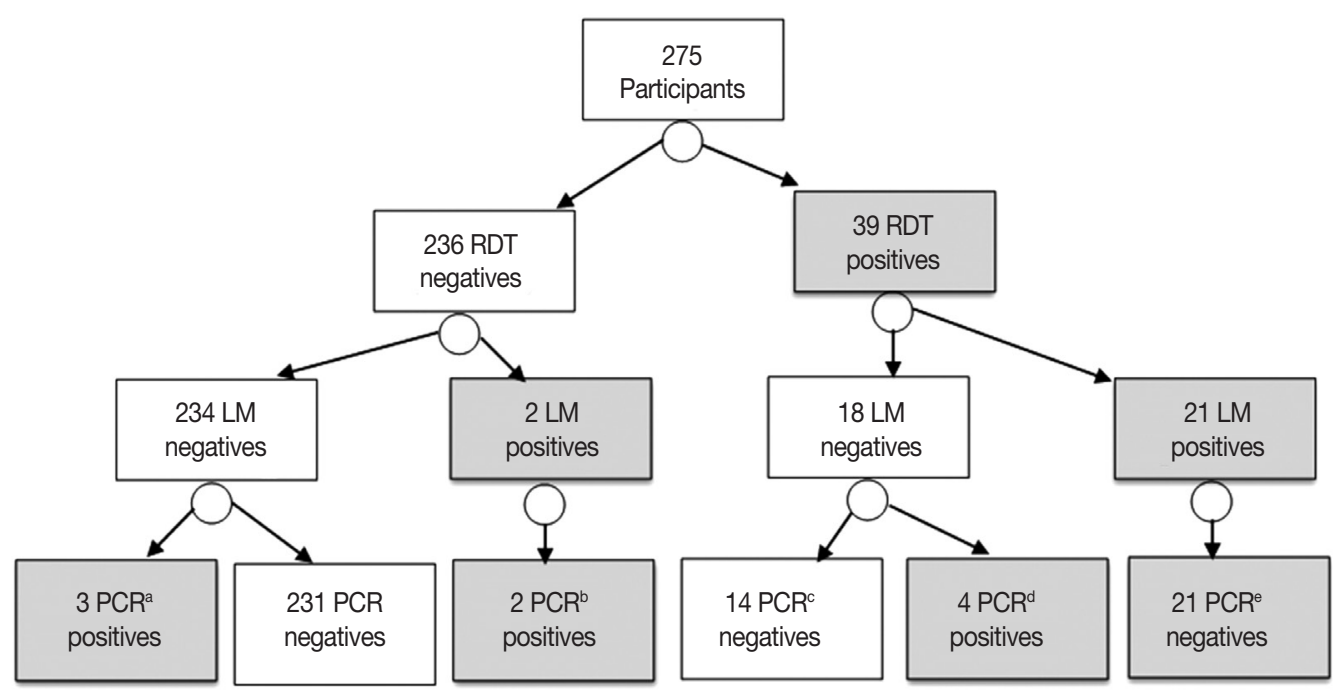

Fig. 4. A flowchart diagram shows results of three tests used for detection and characterization of the malaria cases in this study. RDT (Paramax-3 ${ }^{\text {TM }}$ rapid detection test), LM (light microscopy) and PCR (nested PCR). ${ }^{a} 1$ P. falciparum, 1 P. vivax and 1 mixed infections. ${ }^{b} 2$ P. falciparum infections. ${ }^{c} 10 P$. falciparum and 4 P. vivax. ${ }^{\mathrm{d}} 2$ P. falciparum and $2 P$. vivax. ${ }^{e} 18$ P. falciparum, $2 P$. vivax and 1 as mixed infection.

Table 4. The diagnostic performance of microscopy and the RDT versus the nested PCR

\begin{tabular}{|c|c|c|c|c|c|c|}
\hline \multirow{2}{*}{ Assay } & \multicolumn{3}{|c|}{ Result } & \multicolumn{3}{|c|}{ Diagnostic performance } \\
\hline & Positive No. (\%) & Negative No. (\%) & Se $\%(95 \% \mathrm{Cl})$ & $\mathrm{Sp} \%(95 \% \mathrm{Cl})$ & PPV\% (95\% Cl) & NPV\% $(95 \% \mathrm{Cl})$ \\
\hline Microscopy & $23(8.3)$ & $252(91.7)$ & $76.6(0.57-0.89)$ & $100(0.97-1.00)$ & $100(0.82-1.00)$ & $97(0.93-0.98)$ \\
\hline RDT & $39(14.1)$ & $236(85.9)$ & $83.3(0.64-0.93)$ & $94.2(0.90-0.96)$ & $64.1(0.47-0.78)$ & $97.8(0.94-0.99)$ \\
\hline Nested PCR & $30(10.9)$ & $245(89.1)$ & - & - & - & - \\
\hline
\end{tabular}

$\mathrm{Cl}$, confidence interval; Se, sensitivity; Sp, specificity; NPV, negative predictive value; PPV, positive predictive value; RDT, rapid detection test (Paramax- $\left.3^{\mathrm{TM}}\right) ; \mathrm{PCR}$, polymerase chain reaction.

(1/7), respectively. These sub-microscopic malaria cases were for a patient aged 14 years, three patients 25-44 years and three patients over 44 years, Fig. 2D. Interestingly, the RDT successfully identified four of these sub-microscopic malaria cases: 2 cases were attributed to $P$. falciparum mono-infections and 2 were attributed to $P$. vivax mono-infections.

\section{Paramax- $3^{\text {TM }}$ RDT results}

The RDT identified parasite antigen(s) in 39 patients, with point prevalence of $14.1 \%$ (39/275). The RDT detected the histidine-rich protein 2 antigen in 30 (76.9\%) P. falciparum mono-infections, the lactase dehydrogenase antigen in 8 (20.5\%) P. vivax mono-infections and both antigens in 1 (2.5\%) falciparum/vivax mixed infection. The RDT failed to identify the malaria parasite antigen(s) in 5 (5/30; 16.6\%) patients proved positives by the nested PCR. Furthermore, the RDT assay misdiagnosed 14 patients as malaria positives while the nested PCR identified them as negatives "false positives".
According to the PCR results, these false positives malaria cases were attributed to $P$. falciparum mono-infections in 10 cases and P. vivax mono-infections in 4 cases (Fig. 4).

\section{Diagnostic performance of microscopy and the RDT}

Again, the nested PCR picked 7 malaria cases more than microscopy. Moreover, all the microscopy-positive malaria cases were also positives by the nested PCR. Taking the PCR test results as a nominated gold standard, the smear microscopy exhibited sensitivity (Se), specificity (Sp), positive predictive value (PPV), and negative predictive value (NPV) of $76.6 \%, 100 \%$, $100 \%$, and $97 \%$, respectively (Table 4). By the same principal, the Paramax $-3^{\mathrm{TM}}$ RDT displayed Se, Sp, PPV and NPV of $83.3 \%$, $94.2 \%, 64.1 \%$, and $97.8 \%$, respectively (Table 4).

\section{Concordance of microscopy and the RDT results}

Consistent with the nested PCR results, the light microscopy succeeded to rule out malaria in 14 patients. Moreover, it 
Table 5. Agreement between Paramax-3 $3^{\mathrm{TM}}$ and microscopy tests on 275 patients

\begin{tabular}{|c|c|c|c|c|c|c|c|}
\hline \multirow[b]{2}{*}{ Test } & \multicolumn{3}{|c|}{ Paramax-3 $3^{\mathrm{TM}}$ rapid detection test } & \multicolumn{3}{|c|}{ Agreements (\%) } & \multirow{2}{*}{$\begin{array}{c}\text { Kappa (к) test } \\
95 \% \mathrm{Cl}\end{array}$} \\
\hline & $\begin{array}{l}\text { Positive } \\
\text { (39) }\end{array}$ & $\begin{array}{l}\text { Negative } \\
\text { (236) }\end{array}$ & $\begin{array}{c}\text { Prevalence } \\
\text { (14.1\%) }\end{array}$ & PPA & NPA & ORA & \\
\hline Light microscopy & 23 & 252 & $8.30 \%$ & $53.80 \%$ & $99.10 \%$ & $92.70 \%$ & $\kappa=0.63(0.49-0.78)$ \\
\hline
\end{tabular}

PPA, positive percent agreement; NPA, negative percent agreement; ORA, overall rates of agreement; $\mathrm{Cl}$, confidence interval.

picked up 2 additional $P$. falciparum-positives malarial cases over the RDT. Likewise, the RDT picked up four additional (2 P. falciparum and 2 P. vivax) positive malaria cases over the light microscopy. Taken all together, the overall agreement between the 2 tests were calculated as good (92.7\%; kappa = 0.639) (Table 5).

\section{DISCUSSION}

Prompt diagnosis of malaria in the low transmission settings is important for treatment and infection control. Molecular diagnostic tool capable to detect challenges that arise with the low transmission, is crucial for countries poised to achieve malaria elimination, like Saudi Arabia. In this survey we included a species-specific nested PCR tool into the traditional malaria diagnostics to provide useful insights on several aspects of malaria transmission in Jizan district.

In the current study, the point prevalence of malaria in the study's population was $\sim 11 \%$ with the nested PCR. The vast majority of cases (83.3\%; 25/30) was designated as imported malaria, coinciding with earlier reports. El Hassan and his coworkers have reported high number of imported malaria cases in Jazan [11]. In Hail, another Saudi district, imported malaria has been identified in $25 \%$ of patients based on microscopic examination, 2014-2015 [16]. Notably, the international borders between countries represent a big challenge for countries seeking malaria elimination, as stated elsewhere [17]. In agreement with this observation, the majority of imported malaria cases reported in our study (44\%; 11/25) were originated from Yemen. The Republic Yemen has been frequently considered an important source of imported malaria infections into Saudi Arabia. About 32\% of all imported infections detected in all Saudi area have been arised from Yemen, according to Al Zahrani et al. [18] 2015-2017. The imported malaria sustains potential for indigenous malaria transmission, new Plasmodium species development and emergence of drug resistance [19]. Thus, imported malaria represents a serious threat to infection elimination. In The current study, 16.6\% (5/30) of the detect- ed malaria cases were indigenous. Significant reduction in the number of the locally acquired malaria cases has been exhibited in earlier studies in Jazan and elsewhere in Saudi Arabia. Researchers attributed the reduction of the native malaria cases in the study site to the effective control protocol and to the low rainfall practiced, 2000-2014 [11]. In Taif, a low transmission area, western Saudi Arabia and close to Mecca, much lower estimates of both imported and indigenous malaria have been reported in a hospital-based population [20]. Perhaps, the endemicity pattern of malaria in the study's site, the study's population, the clinical samples and the diagnostic methods used could explain the variation of the imported and indigenous malaria burden among studies.

In the current study, malaria cases were detected throughout the year. Nonetheless, most of cases were identified in February-March and September-October peak periods. This finding might be ascribed to increase travel at these periods. In Aseer, an area near Jazan, the malaria transmission has peaks in February and April following the rains [21]. The local climate and its effect on human-vector activities may have a role for determination of months potentials for peak malaria transmission. Adults aged 15-24 years and males were identified as high-risk groups in the study site, consistent with previous reports $[21,22]$. In our study's location, adult males are more prone to mosquito bite than females and children because they are more likely to be outdoors. We can also point to the fact that most of the migrants who crossed the borders to Jazan, during the study duration, were adult males coming from malaria-endemic areas. In contrast, Jenkins and her co-workers have reported malaria more in females than males in malaria endemic area of Kisumu County, Kenya [23]. This discrepancy may be related to the customs and the traditions of the study's site. In Jazan, by virtue of the customs and the traditions, females wear clothes that cover the entire body and protect them from mosquito bites.

The Plasmodium species distribution in our study was $76.6 \%$ P. falciparum, $16.6 \%$ P. vivax and $6.6 \%$ mixed falciparum/vivax infections. The dominance of $P$. falciparum over $P$. vivax infec- 
tions was evident in our study, consistent with one report [24] and inconsistent with another [16]. Hasona et al. [16] have reported malaria cases attributed more to $P$. vivax than to $P$. falciparum based on RDT. Perhaps, the discrepancy in species distribution between the 2 surveys might be related to the country of the origin of migrants and the Plasmodium species endemicity situation. In our study, the imported malaria cases were primarily originated from $P$. falciparum-endemic countries (Yemen, India, and Sudan) while in Hasona et al. [16], the imported malaria cases have been originated mainly from $P$. vivax-endemic malaria countries (Afghanistan, Pakistan and Iraq). Also, in our study, the nested PCR revealed a low proportion $(0.7 \%)$ of mixed falciparum/vivax infections in the study location. Bin Dajem, in year 2015, has detected a lower proportion (1.9\%) of mixed falciparum/vivax in the study's site [25]. Gupta et al. [26] have reported a much higher proportion (45.5\%) in India. Mixed malaria cases have been overlooked in several Saudi studies, Dawoud et al. [27]'s study is one of these studies. These variations might be due to different methodology adopted in these studies. Mixed malaria infections are often overlooked in studies relying on the classical diagnostic tools. No malaria case attributed to either P. ovale or P. malariae was detected in the study's population, concordant to Dafalla et al. [12].

Approximately $73 \%$ of the detected malaria cases, in our study, were symptomatic (i.e., presence of fever) and $-27 \%$ were sub-patent (i.e., absence of fever). Detection of the subpatent malaria infection is important for the interruption of malaria transmission. The relevance of the symptomless infection rests on the fact that it can transmit malaria in the study's setting [28]. The malaria parasites present in these cases at densities below the limit for microscopic detection and therefore, they were missed detection by the light microscopy. These sub-patent malaria infections were found frequently attributed to $P$. vivax as sole infection species in three cases and concomitant with $P$. falciparum (mixed infection) in one case. Interestingly, out of these eight asymptomatic cases, seven were missed by microscopy (sub-microscopic). The sub-microscopic asymptomatic malaria infections have been demonstrated a contribution to malaria transmission in Jazan [29] and elsewhere in Thailand [30]. These sub-patent infections were most prevalent in patient above 15 years, coinciding with previous report [31]. According to one study, populations with higher levels of immunity (i.e. aged $>15$ years) would maintain the malaria parasites at lower densities enough to be easily missed detection by the microscopy [32].

In this study, we also examined the performance of the three malaria diagnostics. Both the microscopy and the RDT proved less sensitive than the nested PCR, as expected, and already described in a previous study [33]. Microscopy showed the low sensitivity for diagnosis of sub-patent malaria. Seven asymptomatic malaria cases were not recognized by microscopy. Interestingly, four of these sub-microscopic cases were identified by the immunochromatographic test. These four malaria cases might be for patients given anti-malarial treatment and having malaria parasite surface antigens in their blood. The persistent of parasite histidine-rich protein 2 antigen in patients given antimalarial treatment has been previously demonstrated [34]. Consistent with what has been described in the literature [35], the Paramax- $3^{\mathrm{TM}}$ RDT detected malaria infection in $14 \mathrm{pa}-$ tients, not confirmed by the nested PCR. Plasmodium species deficient in the hrp2 gene (e.g. due to deletion) are a possible source of false-negative HRP2 RDTs, according to one study [36]. Of concern, the RDT-false positive results lead to non-essential prescription of the antimalarial drugs that carries side effect on health and may contribute to the emergence of resistant strains [37]. Performance variations of different RDTs have been described based on the transmission settings. Ranadive et al. [38] have attributed the low sensitivity of the RDTs in a hypoendemic malaria setting of Swaziland, to the high proportion of low-density infection among symptomatic patients. On contrast, Kurup and Marks, in a study carried out in Guyana, a high transmission setting, have reported a sensitivity to Paramax- $3^{\mathrm{TM}}$ comparable to that of the microscopic examination [39].

The current study was not free of limitations. Despite of the important data reported in our survey, the survey's data could not apply for the whole district, being a hospital-based. Moreover, our survey's data might be biased by the non-representation of certain population groups in the study's population such as pregnant women, children under 5 years and patients with severe complicated malaria. Also, in the current study, the levels of parasitaemia were not estimated in malaria-positive cases and in our evaluation of the microscopic examination and the RDT, we primarily relied on an assumption taken with the superiority of the nested PCR to the other two tests. Detection of the degree of parasitaemia were not given a consideration our study as the evaluation of the conventional diagnostic tools was not a core purpose of our study. Lastly, the small number of the PCR-confirmed malaria cases made the analysis 
of some collected data very challenging [40].

To conclude, the burden of malaria in Jazan district is relatively high. The largest portion of malaria was imported, largely from Yemen. Infections were attributed mainly to $P$. falciparum and to a lesser extent to $P$. vivax. Despite its relatively low number, falciparum/vivax mixed infections were detected in the study's site. Considerable proportion of asymptomatic sub-microscopic infection was also described. Infections were frequent in adults, males, in patients presented with fever and in patients with recent travel to malaria-endemic areas. Lastly, the low sensitivity of microscopy and the Paramax $-3^{\mathrm{TM}}$ were evident in comparison to the nested PCR. We suggest incorporation of a molecular tool into the traditional malaria diagnosis in Jazan to overcome challenges arising from the low transmission of malaria in the district.

\section{ACKNOWLEDGMENT}

This study was supported by a Research Grant from Deanship of the Scientific Research, Taif University, Saudi Arabia (Research Project No. 1-438-5600).

\section{CONFLICT OF INTEREST}

The authors declare no conflict of interest related to this study.

\section{REFERENCES}

1. World Health Organization. World Malaria Report 2017. Geneva, Switzerland. World Health Organization. 2017.

2. Zhou R, Li S, Zhao Y, Yang C, Liu Y, Qian D, Wang H, Lu D, Zhang H. Characterization of Plasmodium ovale spp. imported from Africa to Henan Province, China. Sci Rep 2019; 9: 2191.

3. Bousema T, Drakeley C. Epidemiology and infectivity of Plasmodium falciparum and Plasmodium vivax gametocytes in relation to malaria control and elimination. Clin Microbiol Rev 2011; 24: 377-410.

4. Bronzan RN, McMorrow ML, Kachur SP. Diagnosis of malaria: challenges for clinicians in endemic and non-endemic regions. Mol Diagn Ther 2008; 12: 299-306.

5. Mathison BA, Pritt BS. Update on malaria diagnostics and test utilization. J Clin Microbiol 2017; 55: 2009-2017.

6. Roth JM, Korevaar DA, Leeflang MM, Mens PF. Molecular malaria diagnostics: a systematic review and meta-analysis. Crit Rev Clin Lab Sci 2016; 53: 87-105.

7. Coleman M, Al-Zahrani MH, Coleman M, Hemingway J, Omar A, Stanton MC, Thomsen EK, Alsheikh AA, Alhakeem RF, McCall
PJ, Al Rabeeah AA. A country on the verge of malaria elimination-the Kingdom of Saudi Arabia. PLoS One 2014; 9: e105980.

8. Al-Zanbagi NA. Review of malaria in Saudi Arabia, current status and future prospects. J Int Acad Res Multidiscip 2014; 2: 1-15.

9. Bousema T, Okell L, Felger I, Drakeley C. Asymptomatic malaria infections: detectability, transmissibility and public health relevance. Nature Rev Microbiol 2014; 12: 833-840.

10. Lin JT, Saunders DL, Meshnick SR. The role of submicroscopic parasitemia in malaria transmission: what is the evidence? Trends Parasitol 2014; 30: 183-190.

11. El Hassan IM, Sahly A, Alzahrani MH, Alhakeem RF, Alhelal M, Alhogail A, Alsheikh AA, Assiri AM, ElGamri TB, Faragalla IA, AlAtas M, Akeel MA, Bani I, Ageely HM, BinSaeed AA, Kyalo D, Noor AM, Snow RW. Progress toward malaria elimination in Jazan Province, Kingdom of Saudi Arabia: 2000-2014. Malaria J 2015; 14: 444.

12. Dafalla OM, Alsheikh AA, Abakar AD, Mohammed WS, Nour BY, Shrwani KJ, Noureldin EM. Identification of Plasmodium species from outdated blood samples by nested-PCR compared with microscopy diagnosis in Jazan region, Saudi Arabia. Biosci Biotech Res Comm 2017; 10: 68-75.

13. Şen Z, Al Alsheikh A, Al-Turbak AS, Al-Bassam AM, Al-Dakheel AM. Climate change impact and runoff harvesting in arid regions. Arab J Geosci 2013; 6: 287-295.

14. Makler MT, Palmer CJ, Ager AL. A review of practical techniques for the diagnosis of malaria. Ann Trop Med Parasitol 1998; 92: 419-433.

15. Isozumi R, Fukui M, Kaneko A, Chan CW, Kawamoto F, Kimura M. Improved detection of malaria cases in island settings of Vanuatu and Kenya by PCR that targets the Plasmodium mitochondrial cytochrome c oxidase III (cox3) gene. Parasitol Int 2015; 64: 304-308.

16. Hasona N, Amer O, Raef A. Hematological alterations and parasitological studies among infected patients with Plasmodium vivax and Plasmodium falciparum in Hail, Kingdom of Saudi Arabia. Asian Pac J Trop Dis 2016; 6: 695-658.

17. Wangdi K, Gatton ML, Kelly GC, Clements AC. Cross-border malaria: a major obstacle for malaria elimination. Adv Parasitol 2015; 89: 79-107.

18. Al Zahrani MH, Omar AI, Abdoon AMO, Ibrahim AA, Alhogail A, Elmubarak M, Elamin YE, AlHelal MA, Alshahrani AM, Abdelgader TM, Saeed I, El Gamri TB, Alattas MS, Dahlan AA, Assiri AM, Maina J, Li XH, Snow RW. Cross-border movement, economic development and malaria elimination in the Kingdom of Saudi Arabia. BMC Med 2018; 16: 98.

19. Al-Farsi HM, Al-Hashami ZS, Dajem SMB, Al-Sheikh AAH, AlQahtani A, Beja-Pereira A, Idris MA, Babiker HA. Source of drug resistant Plasmodium falciparum in a potential malaria elimination site in Saudi Arabia. Infect Genet Evol 2012; 12: 1253-1259.

20. Soliman RH, Garcia-Aranda P, Elzagawy SM, Hussein BE, Mayah WW, Martin Ramirez A, Ta-Tang TH, Rubio JM. Imported and autochthonous malaria in West Saudi Arabia: results from a reference hospital. Malaria J 2018; 17: 286. 
21. Alshahrani AM, Abdelgader TM, Mohya M, Jubran S, Abdoon AMO, Daffalla AA, Babiker A, Kyalo D, Noor AM, Al-Zahrani $\mathrm{MH}$, Snow RW. Risk associated with malaria infection in Tihama Qahtan, Aseer region, Kingdom of Saudi Arabia: 2006-2007. Malaria Contr Elimination 2016; 5.

22. Farag E, Bansal D, Chehab MA, Al-Dahshan A, Bala M, Ganesan N, Al Abdulla YA, Al Thani M, Sultan AA, Al-Romaihi H. Epidemiology of Malaria in the State of Qatar, 2008-2015. Mediterr J Hematol Infect Dis 2018; 10: e2018050.

23. Jenkins R, Omollo R, Ongecha M, Sifuna P, Othieno C, Ongeri L, Kingora J, Ogutu B. Prevalence of malaria parasites in adults and its determinants in malaria endemic area of Kisumu County, Kenya. Malaria J 2015; 14: 263.

24. Al-Zaydani IA, Al-Hakami A, Kumar A, Abdalla SA, Otaif M, Thiqa R, Ahmed H, Alnahili K. Severe unresolving Plasmodium falciparum malaria following artemisinin combination therapy: Emergence of drug resistance in Saudi Arabia. Indian J Med Microbiol 2016; 34: 553.

25. Bin Dajem SM. Molecular investigation of mixed malaria infections in Southwest Saudi Arabia. Saudi Med J 2015; 36: 248-251.

26. Gupta B, Gupta P, Sharma A, Singh V, Dash AP, Das A. High proportion of mixed-species Plasmodium infections in India revealed by PCR diagnostic assay. Trop Med Int Health 2010; 15 : 819-824.

27. Dawoud HA, Ageely HM, Heiba AA. Evaluation of a real-time polymerase chain reaction assay for the diagnosis of malaria in patients from Jazan area, Saudi Arabia. J Egypt Soc Parasitol 2008; 38: 339-350.

28. Manjurano A, Okell L, Lukindo T, Reyburn H, Olomi R, Roper C, Clark TG, Joseph S, Riley EM, Drakeley C. Association of submicroscopic malaria parasite carriage with transmission intensity in north-eastern Tanzania. Malar J 2011; 10: 370.

29. Al-Harthi SA. Comparison of a genus-specific conventional PCR and a species-specific nested-PCR for malaria diagnosis using FTA collected samples from Kingdom of Saudi Arabia. J Egy Soc Parasitol 2015; 45: 457-466.

30. Baum E, Sattabongkot J, Sirichaisinthop J, Kiattibutr K, Jain A, Taghavian O, Lee MC, Davies DH, Cui L, Felgner PL, Yan G. Common asymptomatic and sub-microscopic malaria infections in Western Thailand revealed in longitudinal molecular and serological studies: a challenge to malaria elimination. Malaria J 2016; 15: 333.

31. Whittaker C, Slater HC, Bousema T, Drakeley C, Ghani A, Okell LC. Variation in the prevalence of sub-microscopic malaria in- fections: historical transmission intensity and age as key determinants. bioRxiv 2019; 1: 554311.

32. Steenkeste N, Rogers WO, Okell L, Jeanne I, Incardona S, Duval L, Chy S, Hewitt S, Chou M, Socheat D, Babin FX. Sub-microscopic malaria cases and mixed malaria infection in a remote area of high malaria endemicity in Rattanakiri province, Cambodia: implication for malaria elimination. Malaria J 2010; 9: 108.

33. Mahende C, Ngasala B, Lusingu J, Yong TS, Lushino P, Lemnge $M$, Mmbando B, Premji Z. Performance of rapid diagnostic test, blood-film microscopy and PCR for the diagnosis of malaria infection among febrile children from Korogwe district, Tanzania. Malaria J 2016; 15: 391.

34. Iqbal J, Siddique A, Jameel M, Hira PR. Persistent histidine-rich protein 2, parasite lactate dehydrogenase, and panmalarial antigen reactivity after clearance of Plasmodium falciparum monoinfection. J Clin Microbiol 2004; 42: 4237-4241.

35. Gatton ML, Ciketic S, Barnwell JW, Cheng Q, Chiodini PL, Incardona S, Bell D, Cunningham J, González IJ. An assessment of false positive rates for malaria rapid diagnostic tests caused by non-Plasmodium infectious agents and immunological factors. PLoS One 2018; 13: e0197395.

36. Koita OA, Doumbo OK, Ouattara A, Tall LK, Konaré A, Diakité M, Diallo M, Sagara I, Masinde GL, Doumbo SN, Dolo A. Falsenegative rapid diagnostic tests for malaria and deletion of the histidine-rich repeat region of the hrp2 gene. Am J Trop Med Hyg 2012; 86: 194-198.

37. Shakely D, Elfving K, Aydin-Schmidt B, Msellem MI, Morris U, Omar R, Weiping X, Petzold M, Greenhouse B, Baltzell KA, Ali AS, Björkman A, Mårtensson A. The usefulness of rapid diagnostic tests in the new context of low malaria transmission in Zanzibar. PLoS One 2013; 8: e72912.

38. Ranadive N, Kunene S, Darteh S, Ntshalintshali N, Nhlabathi N, Dlamini N, Chitundu S, Saini M, Murphy M, Soble A, Schwartz A, Greenhouse B, Hsiang MS. Limitations of rapid diagnostic testing in patients with suspected malaria: a diagnostic accuracy evaluation from Swaziland, a low-endemicity country aiming for malaria elimination. Clin Infect Dis 2017; 64: 1221-1227.

39. Kurup R, Marks R. A comparison of microscopic examination and rapid diagnostic tests used in Guyana to diagnose malaria. Malaria Report 2012; 2: e2.

40. Sepúlveda N, Paulino CD, Drakeley C. Sample size and power calculations for detecting changes in malaria transmission using antibody seroconversion rate. Malaria J 2015; 14: 529. 